\title{
Prioridades de pesquisa em enfermagem e as linhas de pesquisa: dando continuidade ao debate
}

\author{
Nursing research priorities and research lines: an on-going discussion \\ Prioridades de investigación en enfermería y las líneas de investigación: dando \\ continuidad al debate
}

\author{
Denize Cristina de Oliveira ${ }^{I}$
}

\begin{abstract}
RESUMO: Objetivou-se apresentar uma proposta de Agenda de Prioridades de Pesquisa em Enfermagem e uma reflexão sobre a importância dessa agenda para a redefinição das linhas de pesquisa. Parte-se da Agenda Nacional de Prioridades de Pesquisa em Saúde e de documentos da Área de Enfermagem para essa proposição, organizada em torno dos seguintes eixos: Saúde, Ambiente, Trabalho e Biossegurança; Avaliação de Tecnologias de Enfermagem e Economia da Saúde; Investigação Clínica; Gestão do Trabalho e Educação em Saúde; Sistemas e Políticas de Saúde; Cuidado à Saúde do Adulto e Idoso; Cuidado à Saúde da Mulher; Cuidado à Saúde da Criança e do Adolescente; Cuidado à Saúde Mental; Cuidado às Doenças Transmissíveis; Cuidado às Doenças Não-Transmissíveis. Conclui-se que as prioridades de pesquisa enfermagem implicam ajustar o foco naquilo que é essencial para dar visibilidade ao saber próprio constituído na área, de forma a definir as facetas disciplinar e interdisciplinar desse campo de conhecimentos.

Palavras-Chave: Prioridades de pesquisa; linhas de pesquisa; investigação; enfermagem.
\end{abstract}

\begin{abstract}
This study aimed at presenting a preliminary Agenda of Research Priorities in Nursing and at debating the importance of this agenda to redefining research lines. This proposal is based on the Agenda of National Priorities in Health Research as well as on nursing-based documents and is organized around the following axes: Health, Environment, Labor and Biosafety; Assessment of Nursing Technology and Health Economics; Clinical Research; Work Management and Health Education; Health Systems and Policies; Adult and Elderly Health Care; Women's Health Care; Child and Adolescent Health Care; Mental Health Care; Communicable Diseases Care; Noncommunicable Diseases Care. Conclusions show that research priorities in nursing imply adjusting the focus to essentials to enhance the specific knowledge in the area so that disciplinary and interdisciplinary limits of this field of knowledge can be defined.
\end{abstract}

Keywords: Research priorities; research lines; research; nursing.

\begin{abstract}
RESUMEN: Este estúdio tuvo como objetivo presentar una propuesta de Agenda de Prioridad de Investigación en Enfermería y una reflexión sobre la importancia de esa agenda para redefinir las líneas de investigación. Esta propuesta fue basada en la Agenda Nacional de Prioridades de Investigación en Salud y en documentos de la área de enfermería, fue organizada en torno a los siguientes temas: Salud, Medio Ambiente, Trabajo y Segurança; Evaluación de Tecnologías de Enfermería y Economía de la Salud; Investigación Clínica; Gestión de Trabajo y Educación en Salud; Sistemas y Políticas de Salud; Cuidado de Salud para el Adulto y el Anciano; Atención a la Salud de la Mujer; Cuidado del Niño y del Adolescente; Atención a la Salud Mental; Cuidado a las Enfermedades Transmisibles; Cuidado a las Enfermedades no Transmisibles. Se concluy que las prioridades de investigación en enfermería implican ajustar el enfoque en lo que es esencial para dar visibilidad a los conocimientos alcanzados para definir los aspectos disciplinarios e interdisciplinarios de ese campo del conocimiento.

Palabras Clave: Prioridades de investigación; líneas de investigación; investigación; enfermería.
\end{abstract}

\section{INTRODUÇÃO}

A preocupação com a temática das prioridades de pesquisa em enfermagem não é nova, mas a continuidade da sua discussão é justificada num momento em que se coloca na pauta da área a necessidade de redefinir as áreas e conhecimento e as linhas de pesquisa orientadoras da produção científica desse campo de conhecimentos. A enfermagem, como campo de saber, ciência e tecnologia vem se fortalecendo desde a década de 70. Sua inserção no Conselho Nacional de Desenvolvimento Científico e Tecnológico (CNPq) ocorreu em 1986 e se consolidou como área específica de produção de conhecimento com o Comitê Assessor de Enfermagem em $2006^{1}$.

A necessidade de definições claras de prioridades e políticas de pesquisa em saúde tem sido destacada no cenário nacional e internacional nos últimos anos. A Política Nacional de Ciência, Tecnologia e Inovação em Saúde (PNCTIS) define a pesquisa em saúde como "o conjunto de conhecimentos, tecnologias e inovações produzidos que resultam em melhoria da 
saúde da população": ${ }^{\text {: }}$. Assim, o desafio da pesquisa em saúde é o de superar uma abordagem disciplinar e caminhar para uma perspectiva setorial e interdisciplinar, incluindo nesse enfoque a totalidade das atividades de pesquisa clínica, biomédica e de saúde pública, além daquelas realizadas nas ciências humanas, sociais aplicadas, e outras que mantenham esta vinculação.

A Agenda Nacional de Prioridades de Pesquisa em Saúde (ANPPS) ${ }^{3}$ é definida como um instrumento de gestão pelo qual o Ministério da Saúde detalha as prioridades de pesquisa para esse campo. Coloca-se como parte fundamental da PNCTIS e objetiva aumentar a seletividade e capacidade de indução dos de fomento à pesquisa no país ${ }^{4}$.

A Agenda Nacional de Prioridades de Pesquisa em Saúde tem como pressuposto respeitar as necessidades nacionais e regionais de saúde e aumentar a indução seletiva para a produção de conhecimentos e bens materiais e processuais nas áreas prioritárias para o desenvolvimento das políticas sociais ${ }^{3}$.

Por meio da ANPPS, as atividades de produção do conhecimento e de fomento devem passar a ser orientadas pelas necessidades de saúde da população, uma vez que abrange toda a cadeia de conhecimentos relacionada à saúde, da pesquisa básica à operacional. Sua elaboração e implementação esteve a cargo do Departamento de Ciência e Tecnologia, um dos três integrantes da Secretaria de Ciência, Tecnologia e Insumos Estratégicos (SCTIE) do Ministério da Saúde".

O presente texto apresenta uma síntese e releitura das exposições realizadas em 2011, no 5o Seminário Internacional dos Núcleos de Pesquisa e Produção do Conhecimento, organizado pela Escola de Enfermagem Anna Nery, e em 2013, no $17^{\circ}$ Seminário Nacional de Pesquisa em Enfermagem ${ }^{5,6}$, e objetivou um resgate dos pontos principais da construção da ANPPS, a atualização dessa discussão para o campo da enfermagem e uma reflexão sobre a importância das prioridades para a redefinição das linhas de pesquisa em enfermagem.

\section{PNCTIS E ANPPS}

A SCTIE assumiu a liderança do esforço nacional de pesquisa em saúde através da definição e da implementação da PNCTIS e da ANPPS. O documento da PNCTIS estabelece que

o Ministério da Saúde deve liderar o processo de construção da Agenda Nacional de Prioridades de Pesquisa em Saúde, em virtude do seu papel estratégico no ordenamento do esforço nacional de pesquisa em saúde ${ }^{2: 3}$.

O texto da ANPPS foi parcialmente aprovado na 2a Conferência Nacional de Ciência, Tecnologia e Inovação em Saúde (CNCTIS) e, posteriormente, referendado pela $151^{\text {a }}$ Reunião Ordinária do Conselho
Nacional de Saúde ${ }^{3}$. Esse processo teve inicio em 2003, quando um grupo designado pelo Conselho Nacional de Saúde propôs 20 subagendas para ter em conta as especificidades da pesquisa em saúde. O segundo passo foi identificar prioridades de investigação para cada subagenda, durante seminários nacionais, envolvendo pesquisadores e formuladores de políticas ${ }^{3,4,7}$.

Durante a fase preparatória da $2^{\text {a }}$ CNCTIS, os municípios e Estados brasileiros organizaram conferências locais, durante as quais foram indicados delegados do setor de saúde. Além desses, foram também designados representantes dos setores da educação e da ciência e tecnologia para participar do debate. Durante a conferência, a ANPPS foi aprovada e quatro outras subagendas foram introduzidas na proposta inicial ${ }^{7,8}$.

A ANPPS é um processo técnico-político em permanente construção e tem como pressuposto atender as necessidades nacionais e regionais de saúde e induzir de forma seletiva a produção de conhecimentos, bens materiais e serviços em áreas estratégicas para o desenvolvimento das políticas sociais em vigor ${ }^{2,3,7}$. O documento é composto de 24 subagendas, que representam as áreas prioritárias de pesquisa. Cada uma dessas subagendas, por sua vez, desdobra-se em temas e linhas de pesquisa, especificando o que é essencial investigar no setor saúde.

Os objetivos que pautaram a Agenda são os seguintes: apontar as principais necessidades nacionais e regionais de pesquisa em saúde, com vistas ao desenvolvimento das políticas sociais; aumentar a seletividade e capacidade de indução de iniciativas de fomento à pesquisa no país; orientar as ações de fomento à pesquisa, permitindo que pesquisadores e financiadores alinhem suas atividades com as exigências nacionais; possibilitar que o governo meça o impacto da pesquisa nacional em saúde; possibilitar que cidadãos informados e organizações da sociedade civil acompanhem os pesquisadores, as instituições de pesquisa e o governo ${ }^{3}$.

Os critérios que pautaram a definição das prioridades compreenderam: carga da doença; análises dos determinantes e da persistência da carga da doença por níveis de intervenção: individual, familiar, comunitário; sistema e serviços de saúde; políticas governamentais e outros setores com impacto na saúde; estado da arte do conhecimento científico e tecnológico disponível; custo-efetividade das possíveis intervenções e possibilidades de sucesso; efeito na equidade e justiça social; aceitabilidade ética, política, social e cultural; possibilidade de encontrar soluções; qualidade científica das pesquisas propostas; factibilidade de recursos humanos e financeiros ${ }^{3}$.

As subagendas definem amplas áreas de pesquisa, envolvendo vários campos disciplinares, que contemplam diferentes temas prioritários de pesquisa e são apresentadas na Figura 13,7: 


\begin{tabular}{|ll|}
\hline 1. Promoção da Saúde & 13. Saúde Mental \\
2. Sistemas e Políticas de Saúde & 14. Alimentação e Nutrição \\
3. Bioética e Ética em Pesquisa & 15. Violência, Acidentes e Trauma \\
4. Comunicação e Informação em Saúde & 16. Saúde, Ambiente, Trabalho e Biossegurança \\
5. Gestão do Trabalho e Educação em Saúde & 17. Doenças transmissíveis \\
6. Saúde do Idoso & 18. Complexo Produtivo da Saúde \\
7. Saúde da Mulher & 19. Assistência Farmacêutica \\
8. Saúde da Criança e do Adolescente & 20. Avaliação de Tecnologias e Economia da Saúde \\
9. Saúde da Pessoa com Deficiência & 21. Investigação Clínica \\
10. Saúde da População Negra & 22. Epidemiologia \\
11. Saúde dos Povos Indígenas & 23. Demografia e Saúde \\
12. Saúde Bucal & 24. Doenças não-transmissíveis \\
\hline
\end{tabular}

FIGURA 1: As 24 subagendas constituintes da Agenda Nacional de Prioridades de Pesquisa em Saúde ${ }^{3,7}$. Brasil,

\section{Prioridades de Pesquisa em Enfermagem}

A enfermagem constitui-se como campo de conhecimento aplicado na grande área da saúde e enquanto tal alinha-se às políticas de saúde de uma forma mais geral. Pensar as prioridades de pesquisa em enfermagem significa apontar focos de interesse e de investimentos, sobre os quais o conjunto de pesquisadores possa estabelecer consensos.

Alguns autores afirmam que

a política de pesquisa em saúde e a Agenda estão atualmente orientando a alocação de recursos do Ministério da Saúde para a investigação e o desenvolvimento científico ${ }^{4: 35}$.

Esses recursos são significativos quando comparados com o montante total dos investimentos realizados pelo Ministério da Ciência, Tecnologia e Inovação. Dessa forma, o alinhamento dos pesquisadores a essa realidade significa, não apenas aderir a uma determinada política, mas também potencializar esforços no sentido da resolução de alguns grandes problemas nacionais de saúde, mobilizando o conhecimento que lhe é próprio.

A temática não é nova na enfermagem, uma vez que o documento Agenda Estratégica para a Pesquisa e Pós-Graduação da Enfermagem Brasileira propõe a

construção coletiva de uma agenda nacional de prioridades de pesquisa em enfermagem, com a seleção de temas e problemas de pesquisa, fundamentada na relação entre as iniciativas das universidades e as demandas sociais ${ }^{9}: 599$.

Esse documento demonstra a preocupação da área de enfermagem com a definição de prioridades de pesquisa, alinhada às discussões do Ministério da Saúde, antes mesmo de ter sido elaborado o documento final da ANPPS.

O estabelecimento de prioridades de pesquisa em enfermagem (PPE) implica em ajustar o foco desse campo de pesquisa naquilo que é essencial para dar visibilidade ao saber próprio constituído, ou seja, no cuidado de enfermagem como categoria teórica, nos sujeitos do cuidado, nas competências profissionais e também nos grandes problemas nacionais transversais, de forma a melhor definir o campo disciplinar e a faceta interdisciplinar desse campo de conhecimentos.

Nesse contexto, partindo da proposição das 24 subagendas e considerando o movimento histórico de desenvolvimento da pesquisa em enfermagem, a autora vem debatendo o tema e apresentando proposições para o reordenação dos campos de investigação da área ${ }^{5,6,10-13}$.

Essas proposições se assentaram nos seguintes passos metodológicos: a partir das 24 subagendas da ANPPS foram selecionados 11 temas afeitos diretamente ao campo de pesquisas de enfermagem; cada um dos 11 temas selecionados foi retraduzido na sua especificidade para o campo da enfermagem; desses 11 temas, cinco caracterizam-se como Eixos Temáticos Transversais, três como Eixos Temáticos de Ações Verticais e outros três como Eixos Temáticos Verticais por Grupos Populacionais; o cruzamento dos cinco eixos transversais e dos seis eixos verticais permite compatibilizar o campo de pesquisa de enfermagem ${ }^{5,6,10,13}$.

As 11 prioridades de pesquisa propostas visam caracterizar o campo de pesquisas da enfermagem e permitir a expressão do saber próprio e da faceta interdisciplinar desse campo. A retradução dessas 11 prioridades de pesquisa na sua especificidade para o campo da enfermagem considerou o foco no cuidado de enfermagem, nos grupos populacionais sujeitos do cuidado, nos grandes problemas nacionais transversais, nas competências profissionais específicas e, ainda, nas temáticas de pesquisa que exigem indução para o seu desenvolvimento.

Essa retradução determinou os cinco Eixos Transversais e os seis Eixos Verticais, que sistematizam prioridades de pesquisa em enfermagem ${ }^{5-6,10,13}$.

Os cinco Eixos Temáticos Transversais são caracterizados a seguir:

1.Saúde, Ambiente, Trabalho e Biossegurança em Enfermagem

2.Avaliação de Tecnologias de Enfermagem e Economia da Saúde

3.Investigação Clínica em Enfermagem

4.Gestão do Trabalho e Educação em Saúde

5.Sistemas e Políticas de Saúde 
Por sua vez, os seis Eixos Verticais subdividem-se em três Eixos Temáticos de Ações Verticais e em três Eixos Temáticos por Grupos Populacionais, definidos a seguir $^{5-6,10,13}$ :

Ações verticais

1.Cuidado de Enfermagem à Saúde Mental

2.Cuidado de Enfermagem as Doenças transmissíveis

3.Cuidado de Enfermagem as Doenças não-transmissíveis

\section{Grupos Populacionais}

1.Cuidado de Enfermagem à Saúde do Adulto e Idoso

2.Cuidado de Enfermagem à Saúde da Mulher

3.Cuidado de Enfermagem à Saúde da Criança e do Adolescente

O cruzamento dos eixos transversais e verticais permite compatibilizar o caráter disciplinar e interdisciplinar característico do campo de pesquisa da enfermagem. Reconhece-se que a clara identificação da faceta disciplinar de um campo de conhecimento é condição essencial para o exercício da interdisplinaridade, resguardando a identidade própria do campo s,6-10-13 $^{\text {. }}$

O debate das linhas de pesquisa na atualidade é premente e vem se expressando em diferentes iniciativas. Uma delas foi o seminário organizado pelo Comitê Assessor de Enfermagem do CNPq em 2013, cujos resultados foram relatados em eventos e publicações ${ }^{11-13}$, traduzindo uma primeira aproximação ao problema. Uma segunda iniciativa foi um estudo desenvolvido para a análise de 617 projetos de pesquisa apresentados ao CNPq, na Área de Enfermagem, nos Editais Universais de 2010, 2011, 2012, cujos resultados são apresentadas no Figura 212:

Como pode ser verificado na comparação das Figuras 1 e 2, as mesmas dimensões temáticas se expressão nos objetos de pesquisa eleitos para os projetos e nas prioridades de pesquisa propostas, variando a maior ou menor agregação das mesmas e a tendência disciplinar ou interdisciplinar observada. Essa constatação pode ser adotada como um indicador de que talvez tenhamos aqui um caminho para uma definição orgânica de prioridades e de linhas de pesquisa.

\section{Conclusões}

A política de pesquisa em saúde e a ANPPS estão atualmente orientando a alocação de recursos do Ministério da Saúde para a investigação, impactando os investimentos brasileiros globais para o desenvolvimento científico, tecnológico e de inovação. Essa constatação exige um posicionamento da enfermagem quanto ao seu alinhamento ou independência em relação à Agenda na definição das suas prioridades de pesquisa e, também, das suas linhas de pesquisa e áreas de conhecimento.

Apresentou-se neste texto a proposição de que as Prioridades de Pesquisa em Enfermagem sejam definidas a partir de paradigmas orientadores como o cuidado de enfermagem, os sujeitos do cuidado, os problemas nacionais transversais, as competências profissionais e os campos de pesquisa que exigem indução para o seu desenvolvimento e fortalecimento, expressando a faceta disciplinar do campo de pesquisa, focada não apenas nos processos mórbidos e nas questões clínicas, mas também na visão integral do processo saúde-doença-cuidado. Por sua vez, as facetas interdisciplinares foram incorporadas através dos eixos temáticos transversais.

Esses paradigmas orientadores exigem o alinhamento das áreas de conhecimento e das linhas de pesquisa em eixos temáticos que permitam expressar a identidade das práticas de pesquisa desse campo, quais

\begin{tabular}{|ll|}
\hline Tipos/Objetos Principais & Objetos Associados \\
\hline Estudos Epidemiológicos-diagnósticos & Adesão aos cuidados \\
& Qualidade de Vida de grupos \\
& Necessidades e Vulnerabilidades de grupos \\
& Políticas de saúde \\
Tecnologias de Enfermagem & Cuidativas \\
& Educativas \\
Cuidado de Enfermagem & De Saúde \\
& Significados \\
Gerenciamento & Cuidado Psicossocial \\
& Grupos populacionais prioritátios \\
Estudos Clínicos & Institucional \\
& Práticas de atendimento \\
& Grupos populacionais \\
\hline
\end{tabular}

FIGURA 2: Objetos de estudo priorizados em projetos de pesquisa apresentados ao CNPq entre 2010 e 2013. Rio de Janeiro, 2014. 
sejam: Saúde, Ambiente, Trabalho e Biossegurança em Enfermagem; Avaliação de Tecnologias de Enfermagem e Economia da Saúde; Investigação Clínica em Enfermagem; Gestão do Trabalho e Educação em Saúde; Sistemas e Políticas de Saúde; Cuidado de Enfermagem à Saúde do Adulto e do Idoso; Cuidado de Enfermagem à Saúde da Mulher; Cuidado de Enfermagem à Saúde da Criança e do Adolescente; Cuidado de Enfermagem à Saúde Mental; Cuidado de Enfermagem as Doenças Transmissíveis; Cuidado de Enfermagem às Doenças Não-transmissíveis.

Destaca-se que o fortalecimento das pesquisas tecnológicas e de inovação apresenta-se como desafio na proposição de processos e produtos do cuidado de enfermagem, buscando melhor definir o desenvolvimento de tecnologias duras, mas também de tecnologias relacionais de cuidado. As dificuldades inerentes ao recorte interdisciplinar do objeto de pesquisa de enfermagem devem ser enfrentadas, articulando-se o processo de cuidar de enfermagem, as suas exigências e condições particulares, permitindo circunscrever um campo de saber próprio, impactando tanto a pesquisa quanto a prática profissional.

Afirma-se, dessa forma, a especificidade da pesquisa e das prioridades de pesquisa em enfermagem no contexto das prioridades de pesquisa em saúde, de forma a permitir a articulação de teorias auxiliares com os paradigmas próprios orientadores desse campo de saber, com vistas à construção de uma ciência da enfermagem, como propõem diversos autores.

\section{REFERÊNCIAS}

1.Erdman AL. Enfermagem Brasileira: avanços na ciência e na tecnologia. Rev Paul Enf. 2006; 25(2):63-5.

2.Ministério da Saúde (Br). Secretaria de Ciência, Tecnologia e Insumos Estratégicos. Departamento de Ciência e Tecnologia. Política nacional de ciência, tecnologia e inovação em saúde. Brasília (DF): Ministério da Saúde; 2005.

3.Ministério da Saúde (Br). Secretaria de Ciência, Tecnologia e Insumos Estratégicos. Departamento de Ciência e Tecnologia. Agenda nacional de prioridades de pesquisa em saúde. Brasília (DF): Ministério da Saúde; 2008. 4.Guimarães R, Santos LMP, Angulo-Tuesta A, Serruya SJ. Defining and implementing a National Policy for Science, Technology, and Innovation in Health: lessons from the Brazilian experience. Cad Saude Publica. 2006; 22:1775-94.

5.Oliveira DC. Políticas da área de enfermagem relativas ao atendimento das prioridades de pesquisa em saúde no Brasil. In: 5 Seminário Internacional dos Núcleos de Pesquisa e Produção do Conhecimento; 2011 out 3-5; Brasil. Rio de Janeiro: EEAN/UFRJ;2011.

6.Oliveira DC. Pesquisa e prioridades de pesquisa em saúde e enfermagem no Brasil. In: Anais do $17^{\circ}$ Seminário Nacional de Pesquisa em Enfermagem; 2013 jun 3-5; Natal, Brasil. Natal (RN): ABEn; 2013.

7.Ministério da Saúde (Br). Departamento de Ciência e Tecnologia, Secretaria de Ciência, Tecnologia e Insumos Estratégicos. Pesquisa em saúde no Brasil. Rev Saúde Pública. 2008; 42:773-5.

8.Ministério da Saúde (Br). Conselho Nacional de Saúde. Conferência nacional de ciência, tecnologia e inovação em saúde. Documento Base. Brasília (DF): Ministério da Saúde; 2004.

9.Associação Brasileira de Enfermagem. Agenda Estratégica para a Pesquisa e Pós-Graduação da Enfermagem Brasileira. Rev Bras Enferm, 2003; 56:599-600.

10. Oliveira DC. Prioridades de pesquisa em saúde e em enfermagem. In: Anais do $17^{\circ}$ Seminário Nacional de Pesquisa em Enfermagem; Natal (RN): ABEn; 2013.

11.Oliveira DC. Áreas de conhecimento do CNPQ: possibilidades e limites. Seminário Nacional de Pesquisa em Enfermagem; Natal (RN): ABEn; 2013. [citado em 10 jun 2014]. Disponível em: http://www.redalyc.org/ articulo.oa? $\mathrm{id}=267028669007$

12. Oliveira DC, Ramos FRS, Barros ALBL, Nóbrega MML. Classification of the CNPq areas of knowledge and the nursing field: possibilities and limits. Rev Bras Enferm. 2013;66(n. esp):60-5.

13. Oliveira DC. Formation pour la recherche et la construction des sciences infirmières au Brésil. In: Jouet $\mathrm{E}$, Las Vergnas O, Noël-Hureaux E, organizadores. Nouvelles coopérations réflexives en santé: de l'expérience des malades et des professionnels aux partenariats de soins, de formation et de recherche. Paris (Fr): Editions des Archives Contemporaines;2014. p.120-38. 